\title{
(Sub-)mm Interferometry in Massive Star Forming Regions
}

\author{
Henrik Beuther \\ Harvard-Smithsonian Center for Astrophysics, 60 Garden Street, \\ Cambridge, MA 02138, USA
}

\begin{abstract}
Sub-)mm interferometry is the most favorable technique to investigate the earliest stages of massive star formation. I will outline general applications in that field and discuss results of different sub-topics (hot core chemistry and massive molecular outflows). Furthermore, recent data obtained with the Submillimeter Array will be shown to present the unique capabilities of this new instrument. Finally, I will give a short outlook on the main physical topics of massive star formation to be tackled with (sub-)mm interferometry within the next decade.
\end{abstract}

\section{Introduction}

As high-mass star formation occurs only in a clustered mode and such regions are on the average at a distance of a few kpc from the sun, high spatial resolution is essential to investigate massive evolving clusters. While more evolved clusters are obvious targets of optical and infrared astronomy, this is different for the earliest stages of their evolution. During their birth-phase high-mass star-forming regions are deeply embedded within their natal cores with column densities as high as $10^{25} \mathrm{~cm}^{-2}$, corresponding to visual extinctions $A_{\mathrm{v}} \sim 10000$. Thus, they are not observable in the near-infrared and even difficult in the mid-infrared (see contribution by J. de Buizer, this volume.). Furthermore, hard X-ray emission as observable with CHANDRA and XMM is only capable of penetrating an $A_{\mathrm{v}}$ of around 100. The other classical approach to investigate massive star-forming regions is studying their free-free emission in the $\mathrm{cm}$ regime. But again, at their earliest stages massive star-forming cores either do not emit free-free emission yet, or they are confined by their surrounding cores that only weak or no free-free emission can escape the region.

However, very young high-mass star-forming regions are strong emitters of cold dust emission which is most prominent in the $\mathrm{mm}$ and sub-mm wavelength regime. In addition, those regions exhibit a rich molecular chemistry which again is best observed in the (sub-)mm regime. Thus, combining the need for high spatial resolution and the strength of young high-mass star-forming regions in the (sub-)mm regime, (sub-)mm interferometry is the best tool to investigate the physical processes in their earliest evolutionary phases. This short review is meant to present the unique capabilities for studying massive star formation with current and future (sub-)mm interferometers. 


\subsection{General applications}

Because (sub-)mm interferometers are sensitive to continuum emission due to the cold dust cores as well as to many molecular line transitions in the (sub-)mm regime, possible fields of research are manyfold. As a very short summary one could categorize some applications:

- Studies of the dust continuum emission to derive masses, column densities, morphologies, multiplicities and eventually a proto-cluster mass function.

- Investigations of the gas dynamics via studying the velocity structure of different molecular line transitions.

- Studying massive proto-stellar disks via continuum and line studies.

- Investigations of the chemical evolution via molecular line studies.

- Studies of massive molecular outflows to get indirect evidence on the physical processes taking place at the core centers.

- Identifications of unambiguous infall signatures via the line-profiles.

Those are not the only applications but these topics cover a wide range of interesting questions.

\section{The hot core $\mathrm{W} 3\left(\mathrm{H}_{2} \mathrm{O}\right)$}

To give an example of gas and dust studies at high spatial resolution, the case of $\mathrm{W} 3(\mathrm{OH})$ is presented. VLA cm studies reveal that $6^{\prime \prime}$ offset from the main UCHII region $\mathrm{W} 3(\mathrm{OH})$ another weaker $\mathrm{cm}$ source associated with $\mathrm{H}_{2} \mathrm{O}$ maser emission is found, dubbed W3 $\left(\mathrm{H}_{2} \mathrm{O}\right.$ ) (Reid et al. 1995, Wilner et al. 1999). Based on the spectral index in the cm regime, Reid et al. (1995) infer that the emission is likely due to a synchrotron jet. Additional VLBI $\mathrm{H}_{2} \mathrm{O}$ maser observations show an outflow around $\mathrm{W} 3\left(\mathrm{H}_{2} \mathrm{O}\right)$ (Alcolea et al. 1992). Wyrowski et al. $(1997,1999)$ observed this source intensely in the $\mathrm{mm}$ regime with the Plateau de Bure Interferometer $(\mathrm{PdBI})$, and their results are quite astonishing. First of all, they detect dust emission associated with the synchrotron jet. Furthermore, they can disentangle two more dust continuum peaks less than 2 arc-seconds apart (Fig. 1a). This indicates that in the close neighborhood of $\mathrm{W} 3(\mathrm{OH})$ another massive cluster is evolving.

In addition to the continuum emission, they observed 6 different molecular species simultaneously. Figure 1 shows three examples of their images. It is striking that nitrogen-bearing species like $\mathrm{HC}_{3} \mathrm{~N}$ are only observed toward $\mathrm{W} 3\left(\mathrm{H}_{2} \mathrm{O}\right)$ - and there only toward the western clump - whereas oxygen-bearing species like $\mathrm{CH}_{3} \mathrm{OH}$ or $\mathrm{SO}_{2}$ are observed toward the young hot core source $\mathrm{W} 3\left(\mathrm{H}_{2} \mathrm{O}\right)$ as well as toward the more evolved UCHII region W3 $(\mathrm{OH})$. Furthermore, based on HNCO observations Wyrowski et al. (1999) estimate gas temperatures toward the $\mathrm{HC}_{3} \mathrm{~N}$ source around $200 \mathrm{~K}$, clearly indicative of a hot core with an internal heating source. The differences in oxygen- and nitrogenbearing molecules are attributed to evolutionary and chemical sequences. Better knowledge of the chemical details will allow using different molecular species as 


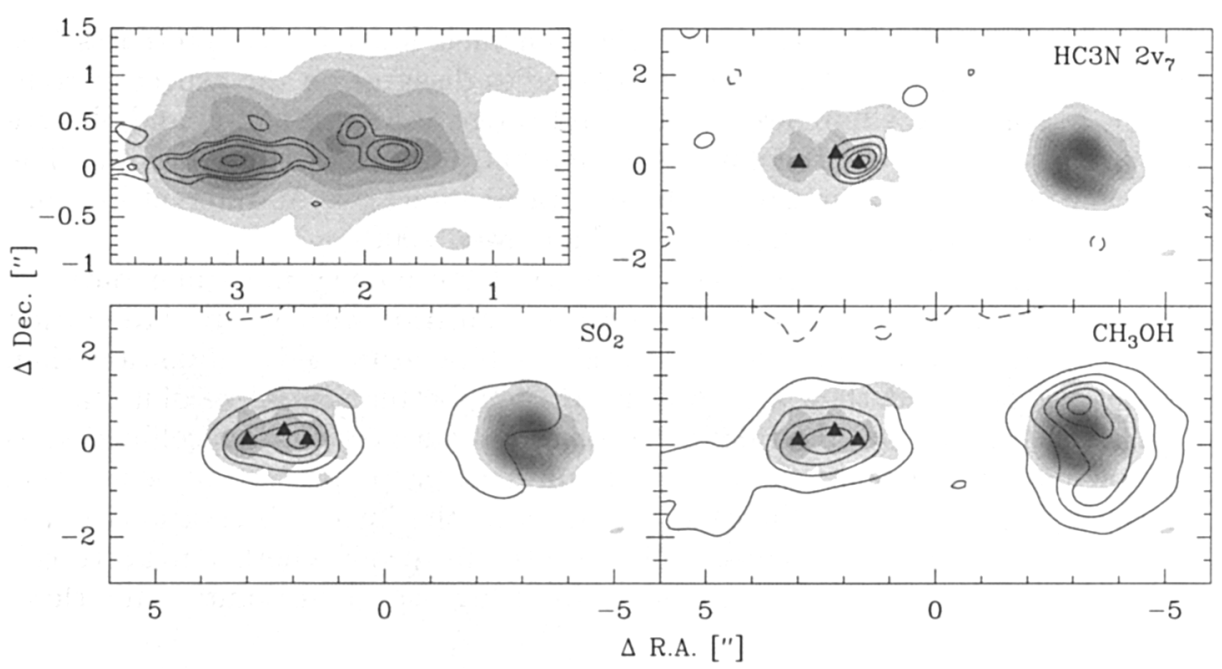

Figure 1. PdBI mm images of W3(OH) by Wyrowki et al. (1999). The top left panel shows a zoom into $\mathrm{W} 3\left(\mathrm{H}_{2} \mathrm{O}\right)$ with the $\mathrm{mm}$ dust continuum in grey-scale and the $\mathrm{cm}$ synchrotron jet in contours (Wilner et al. 1999). The other three panels show the dust continuum emission of the whole region $\mathrm{W} 3(\mathrm{OH})$ in grey-scale and three different molecular transitions as contour overlays. The molecules are marked at the top right of each panel. Triangles show $\mathrm{H}_{2} \mathrm{O}$ masers.

chemical clocks for star-forming regions. Wyrowski et al. (1999) note an additional interesting feature, namely that nearly all oxygen-bearing species peak north-west of $\mathrm{W} 3(\mathrm{OH})$ whereas $\mathrm{SO}_{2}$ peaks slightly east of it (Fig. 1).

\section{Massive molecular outflows}

\subsection{Scientific background}

One of the main questions in massive star formation over the last decade is whether massive stars form in a similar manner as low-mass stars, i.e., via disk accretion processes, or whether different physical processes take place at the very dense evolving core centers, namely the coalescence and merging of intermediatemass proto-stars (e.g., Wolfire et al. 1987, Bonnell et al. 1998, Stahler et al. 2000 , McKee \& Tan 2002). It is still difficult to disentangle high-mass core centers spatially well enough to infer this question in a direct way.

One very promising indirect approach is the observation of massive molecular outflows. The accretion scenario needs massive proto-stellar disks and thus predicts collimated molecular outflows. Contrary, collisions of intermediate-mass proto-stars are presumably extremely energetic and explosive, and it is unlikely that collimated and well ordered structures can survive such processes. Thus, observing molecular outflows, and specifically their degree of collimation, gives a handle on the physical processes taking place at the cluster centers.

Several groups studied massive molecular outflows with single-dish telescopes over recent years (e.g., Shepherd \& Churchwell 1996, Ridge and Moore 2001, Zhang et al. 2001, Beuther et al. 2002a). All those studies agree that massive outflows are ubiquitous phenomena in massive star formation, and that 
they are far more massive and energetic than their low-mass counterparts (see also Richer et al. 2000). However, those studies disagree on the aspect of collimation: Shepherd \& Churchwell (1996) and Ridge \& Moore (2001) and observe on average lower degrees of collimation than known for low-mass outflows. The coalescence community interprets these data as indicative of different entrainment mechanisms (Stahler et al. 2000, Churchwell 2000).

However, Beuther et al. (2002a) observed 26 massive molecular outflows with the IRAM $30 \mathrm{~m}$ telescope in the $\mathrm{CO}(2-1)$ transition with a spatial resolution of $11^{\prime \prime}$. They observe a larger percentage of bipolarity and collimation than previous studies, and they attribute it to their better spatial resolution. To summarize their results: their data are consistent with outflows as collimated as their low-mass counterparts, and no other physical processes need to be invoked.

Nevertheless, even the spatial resolution of the latter observations is too coarse to draw final conclusions, and the ultimate proof whether massive collimated outflows do exist can only stem from high-spatial-resolution interferometric observations.

\subsection{High-resolution observations: The case of IRAS $05358+3543$}

Different groups have embarked on observing massive molecular outflows at high spatial resolution (e.g., Shepherd et al. 1998, Beuther et al. 2002b, Gibb et al. 2003). However, I will focus mainly on the case of IRAS $05358+3543$ observed with the Plateau de Bure Interferometer PdBI (Beuther et al. 2002b). Previous single-dish observations reveal strong wing emission, but determining the outflow morphology is difficult based on the low-resolution data (Beuther et al. 2002a). The picture changes considerably when the source is observed with the high spatial resolution of the PdBI. We observed IRAS $05358+3543$ in two setups, one focused on $\mathrm{CO}(1-0)$ and one observing the $\mathrm{SiO}(2-1)$ and $\mathrm{H}^{13} \mathrm{CO}^{+}(1-0)$ lines simultaneously. Figure 2 presents images of all three lines as overlays on the infrared $\mathrm{H}_{2}$ emission.

The main feature in Figure $2 \mathrm{a}$ is the highly collimated $\mathrm{CO}$ outflow in the east with a collimation degree of 10 , as high as the highest observed in low-mass sources. It emanates from one of three sub-condensations and terminates in the $\mathrm{H}_{2}$ bow shocks in the north and south. The second outflow further west - also in north-south direction - is better depicted by the $\mathrm{SiO}$ data in Figure $2 \mathrm{~b}$. Due to the mass-sensitivity limit around $50 \mathrm{M}_{\odot}$ of the dust continuum emission, no driving source is detected in the mm continuum. Nevertheless, the $\mathrm{H}^{13} \mathrm{CO}^{+}$data show a ridge of three sources in east-west direction. One of those clumps is likely to harbor the driving source of the second outflow. Furthermore, we detect a third outflow inclined by $45^{\circ}$ to the first flow and emanating from the same mmsub-clump (sketched by the two small arrows), likely to harbor a double source. For a more detailed description of the outflow morphologies and dynamics of IRAS 05358+3543 see Beuther et al. (2002b).

The important result one has to take away from those observations is that highly collimated outflows do exist in massive star-forming regions. The largescale eastern outflow is difficult to explain in the coalescence model, whereas it is consistent with a disk-accretion scenario. Furthermore, complicated and seemingly strange features one might observe with single-dish instruments can be disentangled with high enough spatial resolution into features well known from 


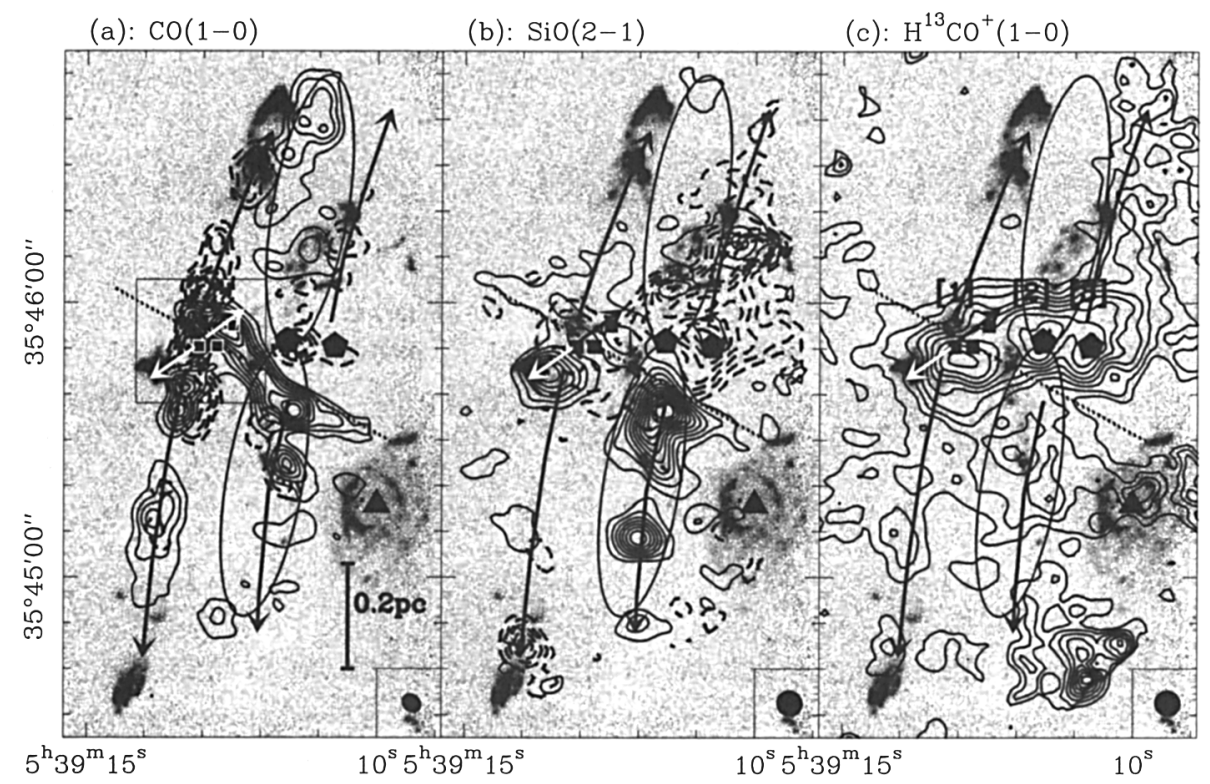

Figure 2. Presented are the PdBI observations of IRAS $05358+3543$ as contour overlays on the grey-scale $\mathrm{H}_{2}$ data. The solid and dashed lines in Figures (a) \& (b) show the blue and red wing emission of the $\mathrm{CO}(1-0)$ and $\mathrm{SiO}(2-1)$ emission, respectively. The solid lines in Figure (c) present the integrated $\mathrm{H}^{13} \mathrm{CO}^{+}(1-0)$ emission. The numbers in brackets label the three $\mathrm{H}^{13} \mathrm{CO}^{+}$sources and the beams are shown at the bottom left. In all images the arrows and ellipses sketch the three outflows (the arrows to the right and the ellipses represent two slightly different interpretations of the western outflow). The three squares represent the three $\mathrm{mm}$ sources, the diamonds locate the $\mathrm{H}^{13} \mathrm{CO}^{+}$peaks, and the triangle marks the IRAS $12 \mu \mathrm{m}$ position.

low-mass star formation. The source IRAS $05358+3543$ is complicated due to its clustered mode of formation and interactions between members of the evolving cluster, but regarding the physical processes we do not observe outstanding differences from low-mass sources. Thus, these data are well consistent with massive stars forming via traditional disk-accretion processes. Beuther et al. (2002b) do not claim that coalescence does not occur, they just infer that it does not seem to be necessary.

The statistical database of high-spatial-resolution outflow observations is still poor but slowly more data emerge which back the case of IRAS $05358+3543$ (e.g., G35.2, Gibb et al. 2003; IRAS 20293+3952, Beuther et al., in prep.).

\section{Recent results from the Submillimeter Array}

As the Submillimeter Array (SMA) is coming online now, I would like to present its first results obtained in the field of massive star formation. The SMA is the first imaging interferometer in the sub-mm wavelength regime, and its capabilities regarding high-mass star formation are unique (Moran 1998). Dust emission scales approximately with $\nu^{4}$, thus the core emission increases quickly when observing at shorter wavelength. Furthermore, massive hot cores are known to 
exhibit molecular line forests (e.g., Schilke et al. 2000), and the broad SMA correlator bandwidth of $2 \mathrm{GHz}$ in a double sideband mode allows one to image a multitude of molecular lines simultaneously. Thus, it is possible to investigate outflows, disks, dust emission, gas dynamics, and chemical processes using the same observations. For more details about the SMA and its recent results see the contributions by Zhang, Ohashi and Wilner in this volume.

In the following, I present recent SMA data observed toward the massive star-forming region IRAS 18089-1732 (Beuther et al., in prep.). This region is in a very early evolutionary stage at the verge of forming a hot core. Figure 3 shows spectra obtained at $217 \mathrm{GHz}$ and $344 \mathrm{GHz}$ with the current bandwidth of $1 \mathrm{GHz}$ (the full $2 \mathrm{GHz}$ will be available within months). Obviously, we observe a forest of molecular lines, and combining the $1 \mathrm{~mm}$ and $850 \mu \mathrm{m}$ data of both sidebands we identify 28 lines from 16 different molecular species.
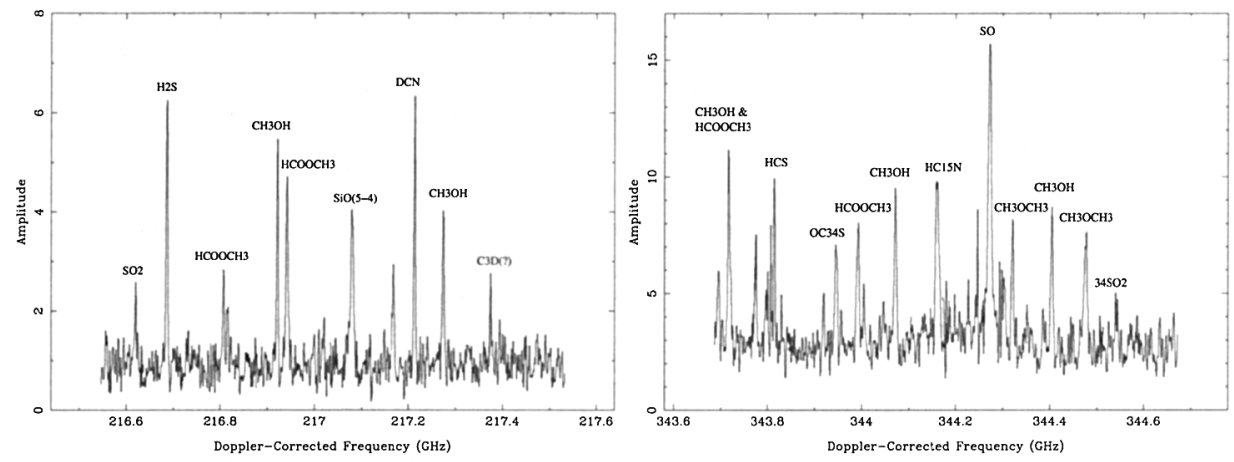

Figure 3. SMA spectra of the high-mass star-forming region IRAS 18089-1732. The left spectrum is taken at $217 \mathrm{GHz}$ and the right one at $344 \mathrm{GHz}$.

Spectra are already interesting, but the excitement of interferometry starts because of its capability to image all those lines simultaneously. Figures $4 \mathrm{a}$ and $4 \mathrm{~b}$ show 2 representative examples of the imaged molecular lines.

The $217 \mathrm{GHz}$ observations were centered at the $\mathrm{SiO}(5-4)$ line to observe jet-like features from this region. Clearly, we identify a collimated outflow emanating in the northern direction from the massive core (Figure 4 left). The dust emission does not split up in multiple sources, but it is resolved and we observe a core-halo structure. Based on the dust emission, the core mass is estimated to be around $1400 \mathrm{M}_{\odot}$.

Figure 4 (right) shows the emission from the high density tracer $\mathrm{HCOOCH}_{3}$ which - as expected - follows the core emission. Interestingly, we find a velocity gradient in east-west direction roughly perpendicular to the $\mathrm{SiO}$ outflow. The spatial scales of that velocity shift are around $3500 \mathrm{AU}$, and we interpret this velocity shift due to the rotation of the outer parts of a disk. A similar velocity pattern is observed in the $\mathrm{CH}_{3} \mathrm{OH}$ line. Disk observations at even higher spatial resolution are crucial to get a more consistent picture of the massive star-forming processes.

Other molecules like $\mathrm{H}_{2} \mathrm{~S}, \mathrm{SO}_{2}$ or DCN show emission toward the core as well as toward the outflow. It is likely that those molecules are released and 

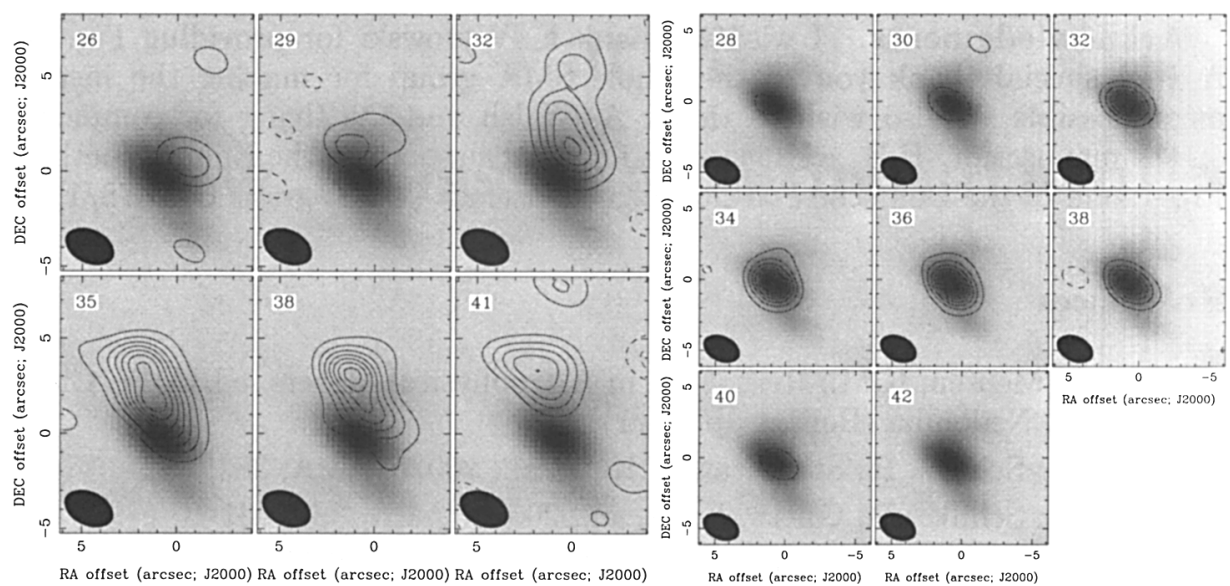

Figure 4. Contour channel maps for IRAS 18089-1732 in the $\mathrm{SiO}(5-4)$ (left) and $\mathrm{HCOOCH}_{3}$ lines (right). The grey-scale shows the $1.3 \mathrm{~mm}$ continuum in all panels.

subsequently processed during shock interactions of the outflow with the surrounding gas.

Already those preliminary results outline the unique capabilities of the SMA, and in the years coming massive star formation research will benefit tremendously from its various applications.

\section{The future of (sub-)mm interferometry}

Millimeter interferometry has proven to be a very powerful tool for investigating the earliest stages of massive star formation. And the future is even brighter. In addition to currently available instruments like the PdBI, NMA and ATCA, the SMA is just at the beginning of scientific research, and last but not least, within the next decade two new instruments will be available, CARMA and ALMA. Especially ALMA with its unprecedented sensitivity, spatial resolution and imaging capabilities will revolutionise not just massive star-forming studies but astrophysical research as a whole.

To conclude, I like to present some scientific topics in high-mass star formation research which will be tackled and hopefully answered within the next decade:

- Detailed studies of massive disks.

- Chemistry of high-mass cores.

- Continuum and IMF studies of massive star-forming regions.

- Absorption and infall studies against the strong dust continuum at very high frequencies.

- Studies of the warm gas within molecular outflows. 
Acknowledgments. I wish to thank F. Wyrowski for providing Fig. 1. A very special thank you to the whole SMA group for making the instrument possible. I also wish to thank A. Walsh and Q. Zhang for comments on the manuscript. H.B. acknowledges financial support by the Emmy-NoetherProgramm of the Deutsche Forschungsgemeinschaft (DFG, grant BE2578/1).

\section{References}

Alcolea, J.; Menten, K.M.; Reid, M.J, in Astrophysical Masers, ed. A.W. Clegg \& G. Nedoluha (Berlin: Springer), 225

Beuther, H.; Schilke, P.; Sridharan, T.K., et al., 2002a, A\&A, 383, 892

Beuther, H.; Schilke, P.; Gueth, F.; et. al., 2002b, A\&A, 387, 931

Bonnell, I.A.; Bate, M.; Zinnecker, H., 1998, MNRAS, 298,93

Churchwell, E., 2000, in: The Origins of Stars and Planetary Systems, eds. Lada C.J. \& Kylafis N.D.

Gibb, A.; Hoare, M.G.; Little, L.T.; Wright, M.C.H., 2003, MNRAS, 339, 1011 Moran, J., 1998, in: SPIE 3357, 208

Reid, M.J.; Argon, A.L.; Masson, C.R.; et al., 1995, ApJ, 443, 238

Ridge, N. A. \& Moore, T. J. T., 2001, A\&A, 378, 495

McKee, C.F. \& Tan, J.C., 2002, ApJ, 416, 59

Richer, J.; Shepherd, D.; Cabrit, S.; et al., 2000, in P \& P IV, ed. V. Mannings

Schilke, P.; Benford, D.J.; Hunter, T.R., et al. 2001, ApJS, 132, 281

Shepherd, D.; Churchwell, E., 1996, ApJ, 472, 225

Shepherd, D.; Watson, A. M.; Sargent, A.I.; Churchwell, E., ApJ, 1998, 507, 861

Stahler S., Palla F., Ho P., 2000, in P \& P IV, ed. V. Mannings

Wilner, D. J.; Reid, M. J.; Menten, Karl M., 1999, ApJ, 513, 775

Wolfire, M.G. \& Cassinelli, J.P., 1987, ApJ, 319, 850

Wyrowski, F.; Hofner, P.; Schilke, P.; Walmsley, C.M.; et al., 1997, A\&A, 320, L17

Wyrowski, F.; Schilke, P.; Walmsley, C.M.; Menten, K.M., 1999, ApJ, 514, L43

Zhang, Q.; Hunter, T.R.; Brand, J.; et al., 2001, ApJ, 522, L167 